\title{
Equilibrium clusters in suspensions of colloids interacting via potentials with a local minimum
}

\author{
A. Baumketner1]; W. $\mathrm{Ca}{ }^{2]}$ \\ ${ }^{1}$ Institute for Condensed Matter Physics, National Academy of Sciences of Ukraine, \\ 1 Svientsistskii St., 79011 Lviv, Ukraine \\ 2 Beijing Computational Science Research Center, Beijing 100094, China
}

Received November 10, 2015, in final form December 18, 2015

\begin{abstract}
In simple colloidal suspensions, clusters are various multimers that result from colloid self-association and exist in equilibrium with monomers.There are two types of potentials that are known to produce clusters: a) potentials that result from the competition between short-range attraction and long-range repulsion and are characterized by a global minimum and a repulsive tail and b) purely repulsive potentials which have a soft shoulder. Using computer simulations, we demonstrate in this work that potentials with a local minimum and a repulsive tail, not belonging to either of the known types, are also capable of generating clusters. A detailed comparative analysis shows that the new type of cluster-forming potential serves as a bridge between the other two. The new clusters are expanded in shape and their assembly is driven by entropy, like in the purely repulsive systems but only at low density. At high density, clusters are collapsed and stabilized by energy, in common with the systems with competing attractive and repulsive interactions.
\end{abstract}

Key words: colloids, clusters, local minimum, repulsive potential, computer simulations

PACS: $64.75 . Y z, 61.20 . G y, 61.20 . J a, 61.20 . N e, 05.10 .-a$

\section{Introduction}

The term "clusters" refers to a large variety of objects that range in size from small multimers to mesoscopic domains [1] and arise as a consequence of monomer self-association in a large variety of soft materials [2]. Most often, clusters are discussed in reference to colloidal suspensions [3], where they exist in equilibrium with monomers, but they were also reported for proteins [4], synthetic clays [5] and metal nanoparticles [6]. Equilibrium clusters become the dominant species in the solution at appropriate thermodynamic conditions. They may also arise transiently, as a consequence of arrested phase transition [7].

As a particular case of the self-assembly process, cluster formation is of key interest to basic research, in particular condensed matter physics. Additionally, it also has appreciable practical applications, for instance as a drug-delivery vehicle [8]. Clusters are capable of significantly altering the mechanical properties of aqueous solutions in which they assemble. This is the case, for instance, of solutions containing monoclonal antibodies, a known biopharmaceutical, which experience a considerable viscosity increase if clusters are present [9]. It is imperative, therefore, to develop a basic understanding of the principles underlying the formation of clusters, in order to use these systems successfully for therapeutic purposes.

Historically, it seems, the possibility of clusters emerging spontaneously in a homogeneous fluid was first raised within the concept of competing interactions. When discussing phase transitions in systems interacting via attractive potentials, Lebowitz and Penrose [10] asked about the effect of an additional repulsive part in the potential, whose range is longer than that of the attractive part. Their conclusion was that the liquid formed as a result of the normal first-order gas-liquid transition due to the attraction among particles would break into finite-size droplets whose size is large compared to the size of the colloid but small compared to the range of the repulsion. Over the past forty years, this scenario was seen to

\footnotetext{
*E-mail: andrij@icmp.lviv.ua
} 
play out in a variety of systems, interacting by a variety of potentials. Kendrick et al. [11] considered a system of colloids interacting via repulsive Coulomb and attractive van der Waals forces and concluded that as a result of the competition between these two interactions, the critical point between the low density liquid phase and the high-density liquid phase is 'preempted by a finite wave vector critical point' [11]. As a result of the new phase transition, macrophases with inhomogeneous density distributions are formed. The same idea of competition between short-range attractive and long-range repulsive forces (SALR) in simple fluids was later researched by Sear et al. [12, 13] in the context of colloids lying on the air-water interface. Analytic mean-field theory [13] and computer simulations [12] indicated that, indeed, instead of the liquid-liquid phase separation (LLPS), colloids form various patterned (modulated) phases, including finite-size clusters. In many respects, these were similar to the patterns created by the competition between repulsion and attraction and described previously for complex fluids [14, 15]. Further progress was made with the work of Groenewold and Kegel [16] who showed how an elaborated model describing the amount of charge on a colloidal surface may lead to the stabilization of clusters of large size. Since that publication, a large amount of work on SALR clusters has followed (see references [17, 18] and references therein) focusing mainly on how the LLPS line is broken/modified by the presence of the repulsion in the potential. A sophisticated and accurate self-consistent Ornstein-Zernike approximation (SCOZA) within the integral-equation theory of the liquid state was used by the group of Reatto [19, 20] to delineate the line in the phase diagram separating the homogeneous fluid phase from the cluster fluid phase, or the so-called $\lambda$-line. The SCOZA predictions were later compared to the results of a densityfunctional theory [21] and simulation [22]. A partial list of other topics that have been covered includes: a) the effect of the attraction range [23, 24], b) the height of the barrier [25], and c) the role of the tail in the potential [26-30].

The second class of systems for which clusters have been reported are colloids interacting via repulsive potentials with a soft shoulder [2]. Stell and Hemmer pointed out in a seminal work [31] that due to the additional length scale, one set by the size of the colloid and the other by the size of the shoulder, such potentials possess very unusual properties. In addition to the usual gas-liquid transition ending in a critical point, for instance, potentials with shoulders may exhibit a liquid-liquid transition, which also ends in a critical point. There have been numerous follow-up studies that focused on the specific details of the new phase transitions as well as on whether or not this model is capable of explaining the thermodynamic anomalies of liquid water [32-36]. It was not until the work of Klein et al. [37] that clusters were made the specific subject of such studies. Using analytical theory and computer simulations, colloids interacting via a hard-core and soft shoulder (HCSS) potentials were seen in this work to form spherical clusters (or clumps) in the fluid phase. At low temperature, clusters were observed to freeze into cluster crystals, cluster glasses and a number of polycrystalline materials [37]. In a later study for the same potential but in 2D, Norizoe and Kawakatsu [38, 39] also reported clusters that are string-like or extended in shape. Extended, chain-like clusters were also observed by Camp [40] for a continuous repulsive potential with a shoulder (different from that of HCSS); the shoulder was determined to be responsible for the emergence of the clusters. In addition to clusters, HCSS are capable of forming a variety of other ordered phases at low temperature. Malescio and Pellicane [41] reported a regular striped phase while Glaser et al. [42] discovered cluster crystals and cluster fluids in MC simulations. Cluster fluids were also reported by Mladek et al. [43], lanes by Fornleitner and Kahl [44], and lamella, hexagonal-columnar and body-center cubic phases, as well as the associated inverse structures by Shin et al. [45]. Dotera et al. [46] describe various quasicrystalline structures that are formed in 2D at low temperature.

It should be noted that many of the properties displayed by the cluster-forming colloids with repulsive interactions actually do not require the presence of the hard core. Likos et al. [47] considered a number of potentials bound at the origin, including the flat portion of the HCSS lacking the hard core, and introduced a criterion for determining if a system with such potential should experience a micro-phase separation into a lattice state where multiple colloids occupy the same lattice site. This criterion was later successfully tested in computer simulations [48], which indeed uncovered cluster crystals. Repulsive [3, 7] as well as attractive [49] colloids were seen to form clusters in laboratory experiments.

In this work we demonstrate by computer simulations that potentials that do not belong to either of the classes mentioned above are also capable of forming equilibrium clusters. Specifically, we consider the potentials that, instead of the global minimum as in the SALR scheme, display a local minimum that has a positive energy and is separated from the longer-distance, zero-energy states by a finite barrier. 
Unlike in the SALR scheme, however, potentials with local attractive minima and long-range repulsion (LALR) lack the energy incentive for the particles to self-associate. LALR model shares with the HCSS potential the global repulsive character and soft shoulder. Experimentally, such potentials may arise as a result of incomplete cancellation between the attractive and repulsive terms. Theoretically, potentials with local minima are little studied. Batten et al. [50] showed that such potentials have unique groundstate configurations, including kagome and honeycomb crystals, and stripes. Liu et al. [51] also examined ground-state configurations of a LALR system confined to a plane. Neither of these papers focused on clusters. To the best of our knowledge, this subject has never been studied.

A comparative analysis of clusters formed by systems of all three schemes, LALR, SALR and HCSS, indicates that the LALR serves as a bridge between the other two. Like in the HCCS model, LALR potentials induce entropy-driven expanded clusters but only at low overall system density and for small cluster sizes. With an increase in the density, the LALR system, like its SALR counterpart, leads to energy driven clusters of large size. The dual identity of the LALR model is also revealed in its temperature behavior. Small clusters of this system are stabilized by temperature, like in the HCSS, but larger clusters are destabilized, in common with the SALR.

\section{Methods}

The shape of the model potential considered in this work is inspired by the inter-molecular potential of aqueous solutions of lysozyme. Using structural functions available from scattering experiments for this protein, effective potentials were derived in our prior work (data not published) for a number of $\mathrm{pH}$ values. For $\mathrm{pH}$ 2, the potential was seen to possess a local minimum at short distances followed by a repulsive term decaying to zero at longer distances. To reflect these features, the following analytical function was considered:

$$
u(r)=\left\{\begin{array}{lll}
+\infty, & r<\sigma_{\mathrm{H}}, \\
-a r+b+\epsilon_{w}, & \sigma_{\mathrm{H}}<r<\frac{\sigma_{\mathrm{H}}+\sigma_{A}}{2}, \quad a=\frac{2 \delta \epsilon}{\sigma_{A}-\sigma_{\mathrm{H}}}, \\
a r-b+\epsilon_{w}, & \frac{\sigma_{\mathrm{H}}+\sigma_{A}}{2}<r<\sigma_{A}, \quad b=\delta \epsilon \frac{\sigma_{A}+\sigma_{\mathrm{H}}}{\sigma_{A}-\sigma_{\mathrm{H}}}, \\
\epsilon_{b} \sigma_{A} \frac{\mathrm{e}^{-\kappa\left(r-\sigma_{A}\right)}}{r}, & \sigma_{A}<r .
\end{array}\right.
$$

The shape of the potential is determined by a set of six basic parameters, $\sigma_{\mathrm{H}}, \sigma_{A}, \epsilon_{w}, \epsilon_{b}, \delta \epsilon$ and $\kappa$. A hardcore wall is placed at $\sigma_{\mathrm{H}}$. The short-range (SA) part of the potential contains a minimum (either local or global) located between $\sigma_{\mathrm{H}}$ and $\sigma_{A}$. The minimum is triangular in shape. Its energy is defined by $\epsilon_{w}$ while the depth is given by $\delta \epsilon$. The long-range part (LR), defined by distances greater than $\sigma_{A}$ is either missing or given by the Yukawa potential characterized by the inverse decay length $\kappa$. The SA and LR parts meet at $r=\sigma_{A}$ where the energy is $\epsilon_{b}$. The schematic of the model potential is shown in figure1 together with its experimental prototype. Depending on the choice of parameters, the potential may represent a number of soft-matter systems. Throughout this article, we use $\sigma_{A}$ as the unit of distance and $\epsilon_{b}$ as the unit of energy. The hard-core diameter is set at $\sigma_{\mathrm{H}}=\sigma_{A} / 3$. The remaining three parameters are varied to achieve the following five representative shapes: 1) Hard-core soft-shoulder (HCSS) potential studied extensively previously in 2- and 3-D [38, 39, 41, 42, 52], 2) Hard-core long-range repulsion potential (HCLR), which, to the best of our knowledge, has not been studied yet in this specific form, but similar potentials were considered in the past [40,53], 3) short-range attraction and long-range repulsion potential (SALR), which was also studied extensively but in a different form [2, 53, 54] , 4) local attraction soft-shoulder (LASS) potential, which has a minimum but no long-range tail and 5) local attractive minimum and long-range repulsion (LALR) potential. The last two potentials are considered here for the first time. The summary of all the employed parameters is shown in table 1

All systems were studied using the standard Metropolis Monte Carlo (MC) method [55]. For a quick scan in the phase space, simulation boxes with 216 particles were considered at 6 values of the total density. The densities ranged from 0.17 to 1.69 in reduced units. A number of temperatures were investigated, depending on the needs of each system, as discussed in the main text. Temperature is reported 


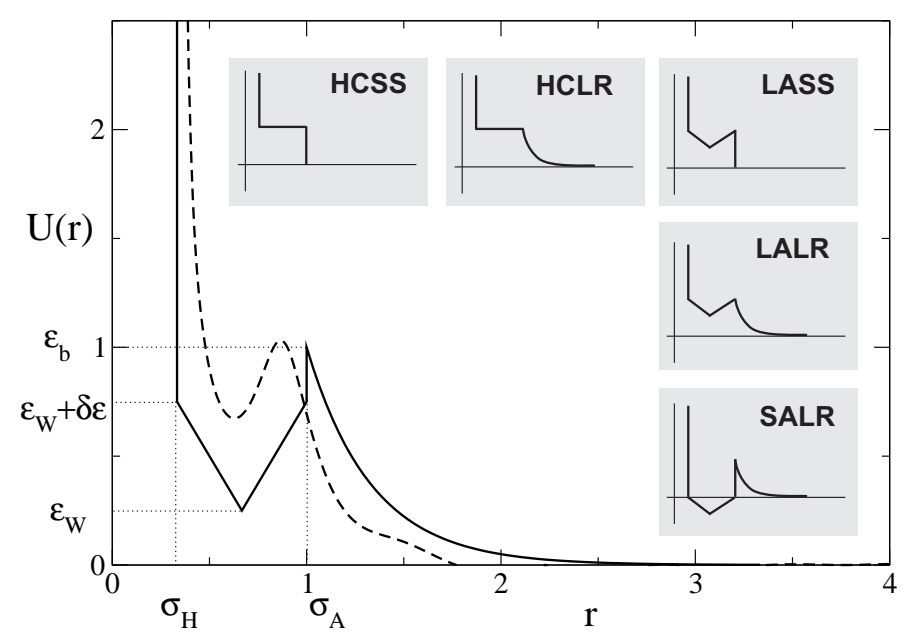

Figure 1. Schematic representation of the potential considered in this work. Depending on the choice of parameters, different types of soft-matter systems can be studied. Five specific shapes investigated in detail are shown as icons. Broken line shows potential, appropriately scaled, obtained for lysozyme solutions at acidic $\mathrm{pH}$.

in units of $\epsilon_{b} / k_{\mathrm{B}}$, where $k_{\mathrm{B}}$ is the Boltzmann constant. In potentials with tails, HCLR, LALR and SALR, the truncation distance $r_{\mathrm{c}}=2 \sigma_{A}$ was used. Tests were performed with longer $r_{\mathrm{c}}$ to make sure that the results are not affected by this parameter. Additionally, simulations with larger boxes containing 5832 particles were conducted for select thermodynamic points in order to extract cluster statistics. The maximum displacement of particles in the MC moves was adjusted to achieve $30 \%$ success rate. All simulations were run for more than $1 \times 10^{6} \mathrm{MC}$ steps and tests were performed to make sure that the reported results are converged.

Table 1. Parameters of five model potentials studied in this work. All energies are measured in units of $\epsilon_{b}$ and all distances in units of $\sigma_{A}$. Abbreviations are as in the main text.

\begin{tabular}{cccc}
\hline \hline Model & $\epsilon_{w}$ & $\delta \epsilon$ & $\kappa$ \\
\hline HCSS & 1 & 0 & \\
HCLR & 1 & 0 & 4.05 \\
LASS & 0.525 & 0.475 & \\
LALR & 0.525 & 0.475 & 4.05 \\
SALR & -0.475 & 0.475 & 4.05 \\
\hline \hline
\end{tabular}

To quantify the process of self-association, all the recorded conformations were clustered. The standard clustering algorithm was used which assigns a particle to the given cluster if its separation from any particle in the cluster is less than a cut-off distance $R_{\mathrm{c}}$. For the cut-off, the diameter of the soft-shoulder $\sigma_{A}$ was used.

In order to characterize the progress of clusterization, a ratio $x$ of the number of particles in monomeric state to the total number of particles was analyzed. Configurations saved in simulations were used to construct a distribution $P(x)$ as a function of density and other thermodynamic parameters. The distribution function yields the free energy profile $\Delta F(x)=-k_{\mathrm{B}} T \log P(x)$, where $k_{\mathrm{B}}$ is the Boltzmann constant and $T$ is the temperature, which has a minimum at the most likely value of $x$. Function $\Delta F(x)$ measures the free energy difference between conformations with two different values of parameter $x$, for instance 0 and 1, in which case it reports the full cost/benefit of converting the system at the given thermodynamic point into clusters. The free energy can be decomposed into internal energy and entropy contributions using the standard thermodynamic relationship $\Delta F(x)=\Delta U(x)-T \Delta S(x)$. The unknown functions $\Delta U(x)$ and $-T \Delta S(x)$ can be determined if the temperature dependence of $\Delta F(x)$ is available. 
First, numerical differentiation of $\Delta F(x)$ can yield $-T \Delta S(x)$ (ignoring the entropy dependence on temperature in the numerical algorithm) and then entropy and free energy can be combined to produce internal energy. This method is commonly used in literature to monitor the progress of various biochemical reactions (see for instance reference [56] and references therein). In the analysis of the free energy profiles, the small size of the simulation box was not seen to affect the conclusions of the work.

\section{Results}

\subsection{All of the studied models form equilibrium clusters}

Several models were studied by computer simulations, as described in detail in the 'Methods' section. All of the studied systems were seen to form equilibrium clusters at appropriate densities and tempera-
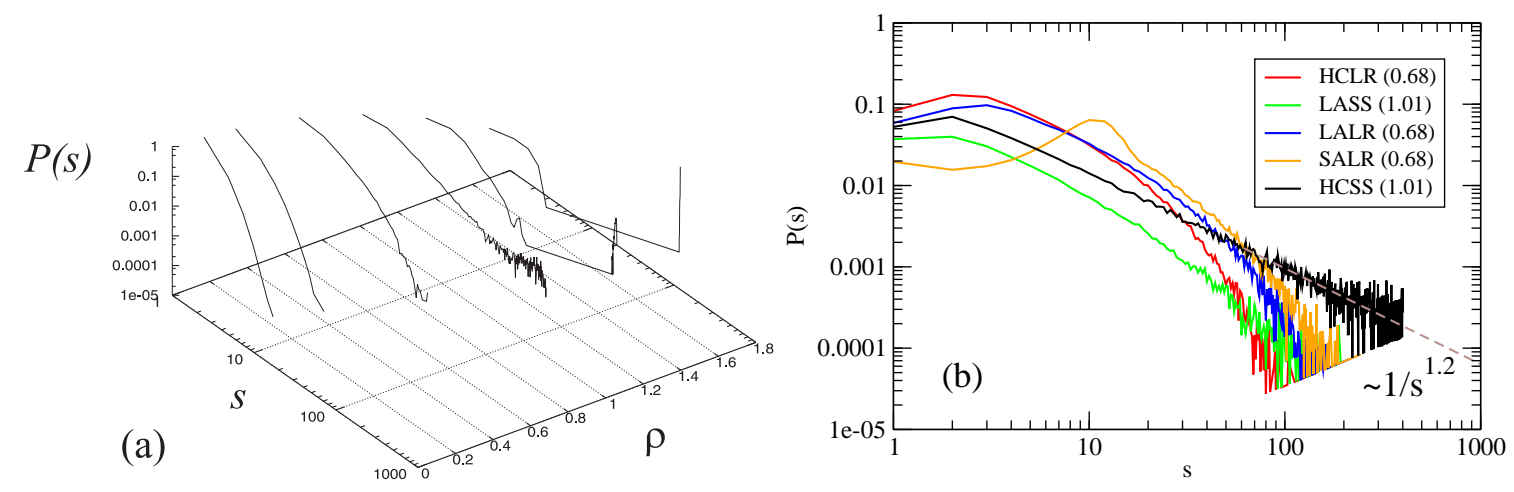

Figure 2. (Color online) The number of particles observed in clusters of specific type relative to the total number of particles in the simulation box. In (a) data for LALR model are shown for select densities. In (b) data for all models are shown, each model at a specific density, indicated between parentheses. All data were collected at a reduced temperature $T=0.62$.

ture. As an illustration, figure 2(a) shows $P(s)$, the ratio of particles engaged in clusters of size $s$ to the total number of particles in the simulation box observed for the HCSS model at a reduced temperature $T=0.62$. At low density $\rho=0.17$ (shown in units of $\sigma_{A}^{-3}$ ), the majority of all particles are monomers. As the density is increased, the proportion of monomers drops at the benefit of dimers, trimers and other multimers. At $0.34<\rho<0.6$, the population of monomers drops below 0.5 , at which point the system becomes dominated by clusters. Clusterization continues for higher densities, and at $\rho=1.01$, the number of monomers drops below the number of particles involved in dimers. At still higher densities, $\rho>1.35$, the system undergoes a percolation transition, above which only a single cluster encompassing the entire simulation box survives. Figure 2(b) shows $P(s)$ recorded for different systems at the density just below the percolation threshold. While all curves demonstrate a majority cluster population, two specific features stand out. First, the SALR simulations show a strong preference for cluster size of 10 monomers. All other systems populate a wide spectrum of sizes with only a small population maximum for dimers and trimers. Second, in the limit of large $s$, the data of HCSS simulations demonstrate a clear power-law dependence, $P(s) \sim 1 / s^{n}$. The observed exponent of $n=1.2$ is in excellent agreement with the prediction of the random percolation limit [39] [note that the usual cluster-size distribution function $n(s)$ is related to the distribution function used here as $n(s)=P(s) / s$ ]. In all other systems, $P(s)$ decays faster.

\subsection{Cluster statistics critically depends on the type of the potential}

All the studied systems populate the clusters that differ widely in size and shape. Radius of gyration $R_{\mathrm{g}}(s)$ is used to characterize how the size of a cluster depends on the number of particles it contains. The most interesting dependence is observed for the SALR potential. Figure 3 shows $R_{\mathrm{g}}(s)$ for this model recorded for several values of the total density of the solution $\rho$. At low densities, the radius of gyration is a monotonous function of the number of particles. Starting at $\rho=0.68$, it begins to develop a plateau, 

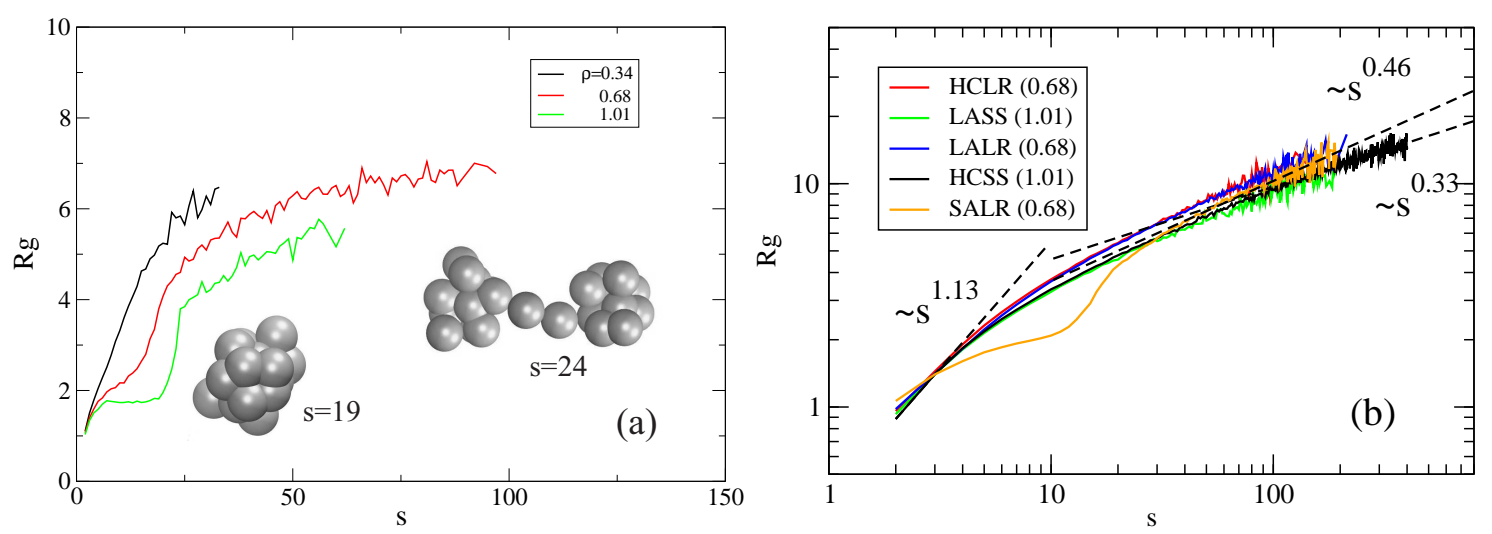

Figure 3. (Color online) Radius of gyration $R_{\mathrm{g}}$ as a function of the number of particles $s$. Data for SALR potential are shown in (a). All potentials with appropriate densities are shown in (b).

signaling the onset of a configurational change. At $\rho=1.01, R_{\mathrm{g}}$ remains unchanged for $3<s<19$ and then jumps twofold for $s>23$. It is easy to figure out what happens at the transition point by examining the shape of the clusters. Representative clusters for $s=19$, which is just before the transition, and $s=24$, which is immediately after it, are shown in figure 3(a). The smaller, or primary, clusters form dense and almost spherical clumps of particles. The larger clusters are made of two (or more) such clumps joined together. Thus, cluster formation is driven by hierarchical supramolecular assembly: small clusters are assembled at initial stages and later on serve as building blocks for larger clusters. This scenario explains the sudden increase in $R_{\mathrm{g}}(s)$ at a certain transition value $s_{\mathrm{c}}$.

The unusual cluster statistics can be rationalized from the standpoint of equilibrium thermodynamics. In common with other SALR potentials [53, 57], the ground-state (GS) configuration of the current model is a 1D Bernal spiral. The energy of the spiral is a monotonously decreasing function of $s$ that continues to decline until all available monomers are absorbed onto the cluster. At zero and sufficiently low temperature, the spiral is the only observable conformation. At finite temperatures, however, this picture changes dramatically. The entropy that is lost by the monomers during the assembly process becomes important. The lowest free energy is achieved through a combination of both internal energy and entropy. The balance between energy and entropy contributions dictates the size of the primary cluster $s_{\mathrm{c}}$. The loss of energy due to the fracturing of the ground state is compensated for by the gain in the entropy arising from the translational freedom of the resulting clusters. As the density of the solution increases (volume is lowered), the entropic gain decreases. So will do the associated loss of energy. As a consequence, the GS conformation will break into a smaller number of pieces. This means that the size of the primary cluster should go up with density. This is exactly what one sees in figure 3 (a) after comparing $R_{\mathrm{g}}(s)$ for $\rho=0.68$ and 1.01 .

The statistics of all other systems are similar to one another and can be described as those of a polydisperse mixture of clusters of different sizes and shapes. As in the case of SALR, the size of the clusters also decreases with the increase of density but this effect is much less pronounced. No non-trivial behavior is seen. A detailed comparison of $R_{\mathrm{g}}(s)$ obtained for different systems [considered at the same densities as in figure 2(b)] is presented in figure 3(b). The plateau in the SALR curve is seen again. Moreover, it is possible to discern the scaling statistics for large clusters. In that limit, the radius of gyration has a powerlaw dependence $R_{\mathrm{g}}(s) \sim s^{n}$ with $n=0.46$, which is independent of the density. This exponent is close to $n=0.5$ observed for ideal, or Gaussian, polymer chains which lack the excluded volume interactions. The Gaussian model mechanism may be applied to the assembly of large colloidal clusters. Although the primary clusters do have the excluded volume, they are allowed to inter-penetrate via the exchange of particles. Thus, from the statistical point of view, they act as zero-size particles. For the same reason, SALR clusters do not experience compression from the environment, as do HCSS clusters, and remain swollen compared to maximally compact states.

The statistics of other systems in the large-cluster limit are governed by $n=0.33$, which corresponds to a maximally compact object. This observation is illustrated in figure 3(b) for HCSS potential. For the 
same system in the limit of small $s$, the radius of gyration scales as $R_{\mathrm{g}} \sim s^{m}$, where $m=1.13$. Note that for the linear arrangement of the smallest clusters, dimer and trimer, the exponent $m=1.2$. Therefore, HCSS behaves as a linear chain for small $s$. This is in agreement with the prior work on this system [39], which also shows that the linear scaling persists to large clusters for a specific choice of potential parameters (different from those of the present work). HCLR, LASS and LALR potentials also exhibit almost a linear scaling but with the exponent that is system-specific. In common with the HCSS model, clusters formed by these potentials experience a cross-over from a linear regime for small clusters to a collapsed chain for large clusters. The transition is gradual and takes place over two orders of magnitude. This behavior is in sharp contrast to the SALR model and can be explained by the specific character of the HCSS interaction. Although the formation of clusters in this model is driven by entropy (see below), the specific shape of different clusters of the same size is also influenced by energy. Since the interaction is purely repulsive, particles within a cluster will choose to reside as far from their neighbors as possible. This results in linear conformations for small clusters, which minimize the overlap between constituent particles. For larger clusters, the minimal overlap can be achieved in string-like but bended conformations, similarly to those observed in the self-avoiding polymers. Like in the polymers, colloidal clusters should experience an entropic collapse with the pertinent exponent of the radius of gyration of $m=0.591$, or approximately $3 / 5$ as predicted by Flory [58]. The fact that the observed exponent 0.33 is lower indicates that large clusters experience additional compression from the environment, which may only result from the repulsive interactions of clusters with other species in the solution, both clusters and monomers alike. These theoretical arguments lead us to the conclusion that the specific details of the cross-over will depend on a) the extent of the soft shoulder in the potential, which governs how much linear chains gain in energy compared to the collapsed ones and b) the strength of the repulsive tail, which determines how strongly various colloidal species repel each other, thus inducing cluster collapse.

\section{Discussion}

\subsection{Two classes of cluster-forming systems use different assembly mechanisms}

Our simulations clearly demonstrate that all of the studied systems form equilibrium clusters. In view of this finding, the relevant question to ask is: 'Why does that happen? What are the pertinent mechanisms?'. Perhaps the simplest to explain is the mechanism of the SALR model. By design, this potential favors association of particles by providing a negative potential energy to configurations with close separation among particles. Clusters of different topologies represent a ground-state configuration of SALR potentials [2, 57], including the one studied here. Thus, for these systems, cluster assembly is expected to be driven by internal energy. To quantify this assessment, it makes sense to analyze the free energy profile of the clusterization reaction as a function of a certain order parameter. Since we are interested in the transition into the cluster state in general, not into a specific type of cluster, it is most convenient to monitor the number of particles in the monomeric state as a progress variable. To make a comparison between systems of different sizes easier, the number of monomers will be normalized by the total number of particles, producing a ratio of monomers $0<x<1$. Conformations with no clusters have $x=1$ while those lacking monomers (complete transformation into clusters) are characterized by $x=0$. Internal energy, $\Delta U(x)$, and entropy, $-T \Delta S(x)$, can be computed for each $x$ (see 'Methods' section) to elucidate the role of these functions in the cluster formation. Figure 4 (a) shows $\Delta U(x)$ and $-T \Delta S(x)$ obtained for the SALR model at $\rho=0.68$. As predicted above, the cluster formation is driven by energy and opposed by entropy. The two curves combined yield a minimum of free energy at about $x=0.015$.

The same analysis was repeated for the HCSS potential. Note that this model in 2D, given the appropriate choice of parameters, forms ordered configurations in the ground and low-energy states [59] as well as quasi-crystals [46]. Thus, one may expect the remnants of ordered structures to survive at finite temperature, giving rise to clusters stabilized by energy [42]. Figure 4(b), depicting $\Delta U(x)$ and $-T \Delta S(x)$ demonstrates that this expectation is not justified. Clusters are stabilized entirely by entropy and destabilized by energy. The two systems, SALR and HCSS, differ dramatically in their cluster formation scenarios. While clusters in SALR tend to stay intact due to the mutual attraction, clusters in the HCSS model use a different mechanism. Namely, particles at distances $\sigma_{\mathrm{H}}<r<\sigma_{A}$ experience no force and move freely 

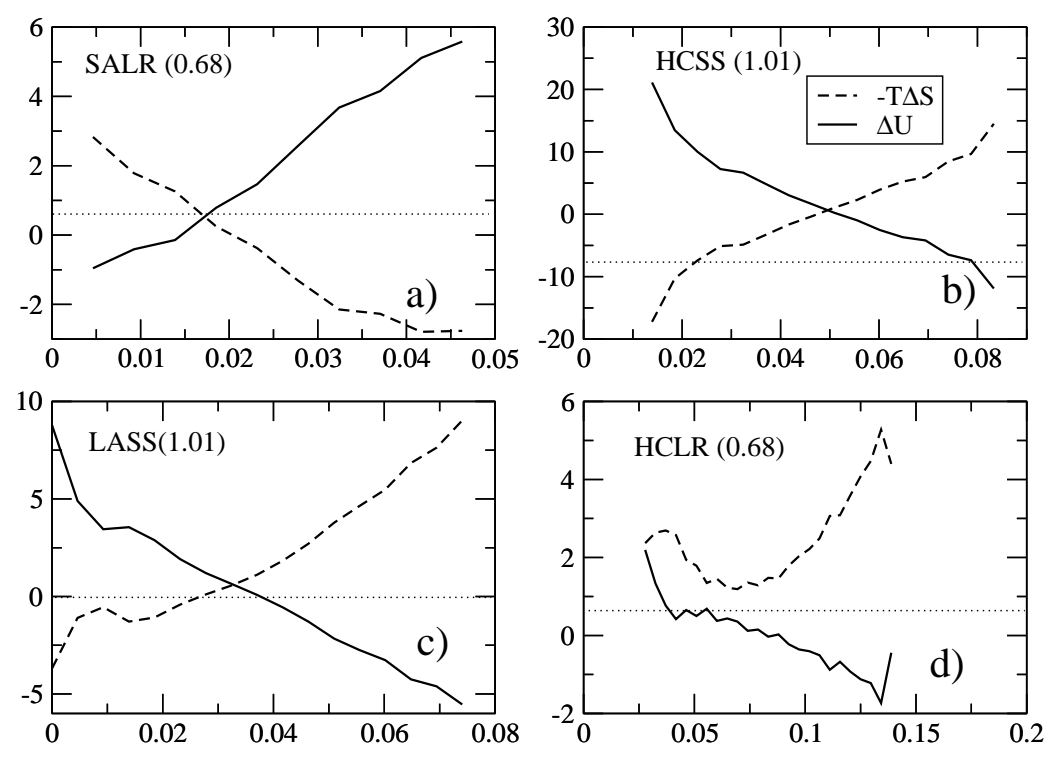

$\mathrm{X}$

Figure 4. Internal energy, $\Delta U(x)$, and entropy, $-T \Delta S(x)$, as a function of the fraction of monomeric particles $x$ generated in this work for different cluster-forming potentials. All models except SALR exhibit the clusters stabilized by entropy. Here, and elsewhere in the article, energy is measured in units of $\epsilon_{b}$.

about each other. Particles in the monomeric state experience no force either, since the potential is zero for $r>\sigma_{A}$. Therefore, clusters stay together because the volume created by joining the soft-shoulder regions of all constituent particles is greater than the volume available to them in the monomeric state (at a distance $r>\sigma_{A}$ from any other monomer or cluster). This point is illustrated in figure 5 For a monomeric particle, the moment of joining an existing cluster is accompanied by an increase in energy as well as by an increase in entropy. As long as the entropy wins, particles spend the majority of their time in cluster configurations. Note that these clusters will have a very short residence time of the order of the duration of binary collisions. They will also rapidly disintegrate in the event of an increase in the available volume.

Cluster formation in HCRL and LASS models is driven by the same mechanism as in the HCSS model. Adding a repulsive tail to the potential, as in HCLR, changes the appearance of $-T \Delta S(x)$ : at small $x$ it

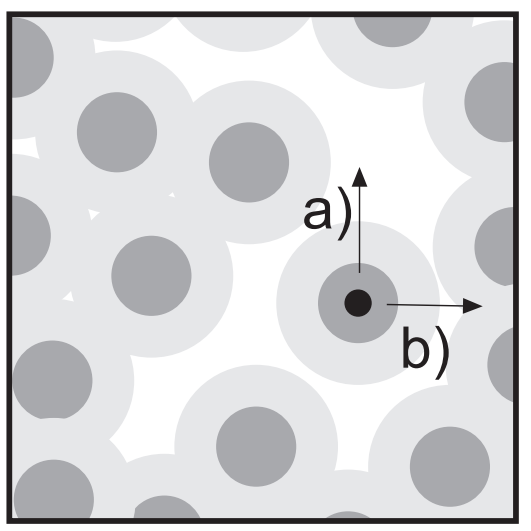

Figure 5. Illustration of how entropy stabilizes the clusters in the HCSS model. The central monomeric particle, denoted by black circle, has the choice of either to: a) remain monomeric, in which case it is allowed to explore white-colored space, or b) join a cluster, in which case it is allowed to explore lightgray space. Dark gray space indicates hard-core areas which are not accessible to the particle. Clusters become stable when the volume of the light-gray area exceeds that of the white area. 
begins to disfavor clusters, see figure 4 (d). However, the destabilizing effect of energy is unaffected. An additional minimum in the soft shoulder, as in LASS, decreases the destabilizing effect of energy, compare figure 4 (c) and (b), but not to the extent of reversing its role. Cluster formation of both systems is driven by entropy.

\subsection{The model with local minimum exhibits a dual identity}

The model with the local minimum and repulsive tail stands out from the rest of the studied systems. Figure 6 shows its energy and entropy evaluated at two different densities, $\rho=0.34$ and $\rho=0.68$. At a lower density, the mechanism is as in the HCSS system with the entropy stabilizing the clusters. For a higher density, the mechanism changes and becomes similar to that of the SALR potential, where the internal energy drives the assembly of clusters. The transformation is not complete as entropy still favors the clusters at high density instead of disfavoring them, as in the SALR potential. Nevertheless, the reversal of the energy role is quite remarkable. This is not seen in any other potential and indicates that the process of cluster formation in the systems interacting via potentials with local minima can be extraordinarily complex. It takes the presence of both monomers and small clusters in such systems for the clusterization to be driven by energy. Any theory that aims at predicting the cluster distribution quantitatively should be capable of capturing that effect. That means a proper description of monomer/cluster mixture, including the association/dissociation balance, across a wide range of densities. It is not clear which of the currently available liquid-state theories would be capable of accomplishing this task.

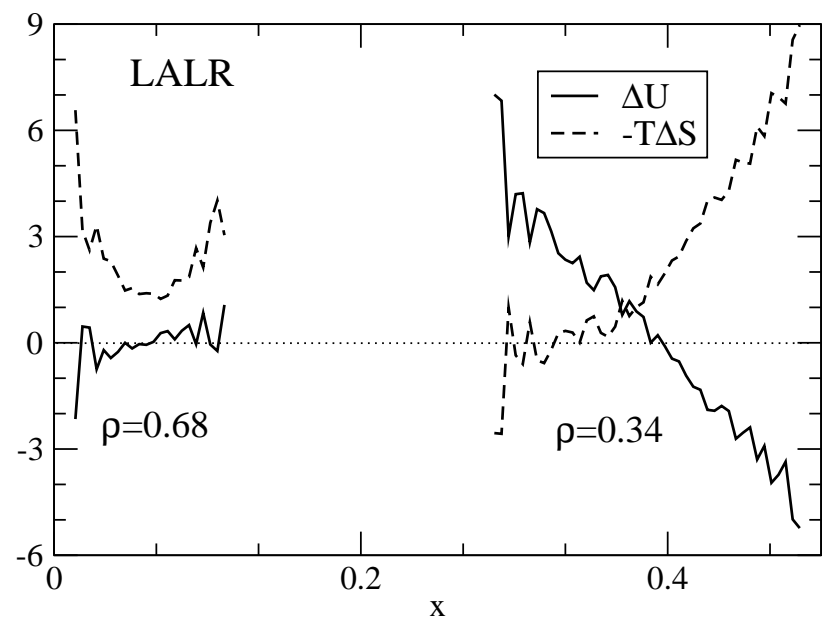

Figure 6. The same as figure 4 but for the potential with local minimum and repulsive tail, LALR. Both entropy- and energy driven mechanisms of cluster formation are present, depending on the total density of the solution.

The split identity of the LALR model is also evident in its temperature behavior. Since the assembly of clusters in the HCSS potential is entropic in nature, it should be enhanced by temperature. The opposite is true for the SALR model, where clusters should become less stable at higher temperature. Figure 7 showing the fraction of monomers as a function of temperature and density for different models, confirms this conjecture. Different symbols in this figure represent different systems, broken lines correspond to a high temperature of 0.62 while solid lines stand for the low temperature of 0.21 . For the HCSS model, the population of monomers at the lower temperature is higher than the population at a higher temperature, the solid line is above the broken line, at all densities, indicating that cluster formation is enhanced by temperature. For the SALR model, the broken line is above the solid line, which is the evidence that temperature hinders the formation of clusters. For the LALR potential, the broken line is above the solid line for $\rho<0.68$ and vice versa for higher densities. Thus, this model exhibits the features characteristic of both classes of cluster-forming colloids. The entropy-driven mechanism dominates at low densities while the energy-driven mechanism - at high densities. 


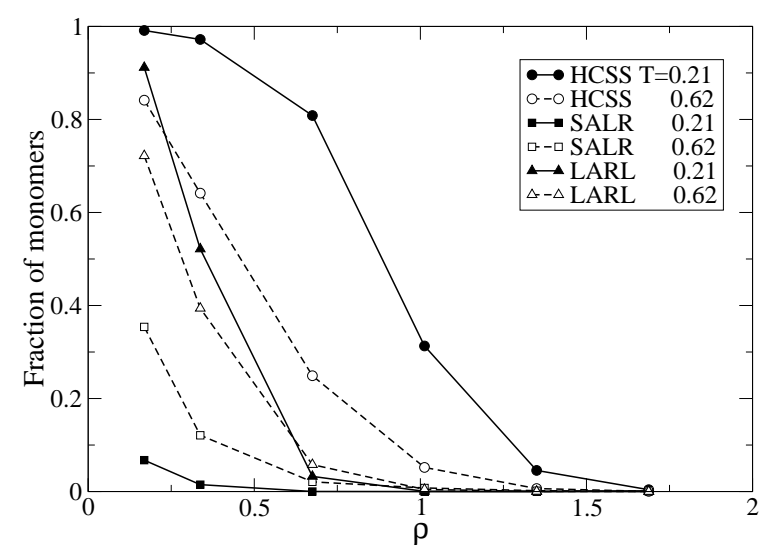

Figure 7. Fraction of monomers in the conformational ensemble obtained for different models, temperatures and densities. HCSS clusters are destabilized by temperature. SALR clusters are stabilized by temperature while LALR clusters exhibit both behaviors depending on density.

\section{Conclusions}

There are two known types of cluster-forming colloids: a) those that interact via purely repulsive potential with a soft shoulder and, possibly, a tail, and b) those that are characterized by a potential with the global attractive well at short distances followed by a repulsive tail at long distances. By adding an attractive term to the otherwise repulsive potential, it is easy to transform the potentials of the first type into the potentials of the second type. Thus, it may seem obvious that systems of the two types exhibit similar properties, in particular the capability of forming clusters. However, there is one caveat with this argument. Extrapolating properties of one system based on the results of another system makes sense only if the two are sufficiently similar so that perturbation theory can be used. This is clearly not the case for the cluster-forming colloids. Repulsive and attractive potentials exhibit completely different structural, dynamic and phase behaviors. Take, for instance, the gas-liquid transition in attractive colloids that is missing in the repulsive colloids. The differences also extend to the clusters of these systems. While attractive potentials form compact clusters of specific size, the repulsive potentials lead to extended, polydisperse multimers. There is only a limited amount of information that can be learned about one system by extrapolating the properties of the other. Simulations remain the most reliable and accurate tool in the studies of a new or unknown potential. Simulations played a key role in this work, helping us to reveal that a repulsive potential with a local minimum shares certain properties with both other types of cluster-forming potentials. The clusters it populates are similar to those of purely repulsive potentials, while the energy-driven mechanism is similar to the attractive potentials.

Our results were obtained for a specific shape of the potential with a local minimum. How general they are with respect to other systems remains to be seen. Of particular interest here would be to consider square-well potentials which have been studied extensively in the literature in regard to phase transitions. Also, of interest would be to examine the behavior of continuous potentials, like those that can be constructed with the help of two Yukawa functions.

\section{References}

1. Vekilov P.G., Rev. Chem. Eng., 2011, 27, No. 1-2, 1; doi 10.1515/revce.2011.003

2. Dinsmore A.D., Dubin P.L., Grason G.M., J. Phys. Chem. B, 2011, 115, No. 22, 7173; doi $10.1021 / j p 202724 b$

3. Osterman N., Babic D., Poberaj I., Dobnikar J., Ziherl P., Phys. Rev. Lett., 2007, 99, No. 24, 248301; doi 10.1103/PhysRevLett.99.248301

4. Stradner A., Sedgwick H., Cardinaux F., Poon W.C.K., Egelhaaf S.U., Schurtenberger P., Nature, 2004, 432, No. 7016, 492; doi 10.1038/nature03109

5. Atmuri A.K., Bhatia S.R., Langmuir, 2013, 29, No. 10, 3179; doi 10.1021/la304062r 
6. Tam J.M., Murthy A.K., Ingram D.R., Nguyen R., Sokolov K.V., Johnston K.P., Langmuir, 2010, 26, No. 11, 8988; doi 10.1021/la904793t

7. Janai E., Schofield A.B., Sloutskin E., Soft Matter, 2012, 8, No. 10, 2924; doi 10.1039/C2SM06808G

8. Xu S., Sun C., Guo J., Xu K., Wang C., J. Mater. Chem., 2012, 22, No. 36, 19067; doi 10.1039/C2JM34877B

9. Yearley E.J., Godfrin P.D., Perevozchikova T., Zhang H.L., Falus P., Porcar L., Nagao M., Curtis J.E., Gawande P., Taing R., Zarraga I.E., Wagner N.J., Liu Y., Biophys. J., 2014, 106, No. 8, 1763; doi 10.1016/j.bpj.2014.02.036

10. Lebowitz J.L., Penrose O., J. Math. Phys., 1966, 7, No. 1, 98; doi 10.1063/1.1704821.

11. Kendrick G.F., Sluckin T.J., Grimson M.J., Europhys. Lett., 1988, 6, No. 6, 567; doi 10.1209/0295-5075/6/6/016

12. Sear R.P., Chung S.W., Markovich G., Gelbart W.M., Heath J.R., Phys. Rev. E, 1999, 59, No. 6, R6255; doi 10.1103/PhysRevE.59.R6255

13. Sear R.P., Gelbart W.M., J. Chem. Phys., 1999, 110, No. 9, 4582; doi 10.1063/1.478338

14. Wu D., Chandler D., Smit B., J. Phys. Chem., 1992, 96, No. 10, 4077; doi $10.1021 / j 100189 a 030$

15. Seul M., Andelman D., Science, 1995, 267, No. 5197, 476; doi $10.1126 /$ science.267.5197.476

16. Groenewold J., Kegel W.K., J. Phys. Chem. B, 2001, 105, No. 47, 11702; doi 10.1021/jp011646w

17. Zhang T.H., Kuipers B.W.M., Tian W.D., Groenewold J., Kegel W.K., Soft Matter, 2015, 11, No. 2, 297; doi 10.1039/C4SM02273D

18. Sweatman M.B., Fartaria R., Lue L., J. Chem. Phys., 2014, 140, No. 12, 124508; doi 10.1063/1.4869109

19. Pini D., Ge J.L., Parola A., Reatto L., Chem. Phys. Lett., 2000, 327, No. 3-4, 209; doi 10.1016/S0009-2614(00)00763-6

20. Pini D., Parola A., Reatto L., J. Phys.: Condens. Matter, 2006, 18, No. 36, S2305; doi 10.1088/0953-8984/18/36/S06.

21. Archer A.J., Pini D., Evans R., Reatto L., J. Chem. Phys., 2007, 126, No. 1, 014104; doi $10.1063 / 1.2405355$

22. Archer A.J., Wilding N.B., Phys. Rev. E, 2007, 76, No. 3, 031501; doi 10.1103/PhysRevE.76.031501

23. Charbonneau P., Reichman D.R., Phys. Rev. E, 2007, 75, No. 1, 011507; doi 10.1103/PhysRevE.75.011507

24. Tarzia M., Coniglio A., Phys. Rev. E, 2007, 75, No. 1, 011410; doi 10.1103/PhysRevE.75.011410.

25. Costa D., Caccamo C., Bomont J.M., Bretonnet J.L., Mol. Phys., 2011, 109, No. 23-24, 2845; doi $10.1080 / 00268976.2011 .611480$

26. Groenewold J., Kegel W.K., J. Phys.: Condens. Matter, 2004, 16, No. 42, S4877; doi 10.1088/0953-8984/16/42/006

27. Imperio A., Reatto L., J. Phys.: Condens. Matter, 2004, 16, No. 38, S3769; doi 10.1088/0953-8984/16/38/001

28. Hutchens S.B., Wang Z.G., J. Chem. Phys., 2007, 127, No. 8, 084912; doi $10.1063 / 1.2761891$.

29. Liu Y., Chen W.R., Chen S.H., J. Chem. Phys., 2005, 122, No. 4, 044507; doi $10.1063 / 1.1830433$

30. Schwanzer D.F., Kahl G., J. Phys.: Condens. Matter, 2010, 22, No. 41, 415103; doi 10.1088/0953-8984/22/41/415103

31. Hemmer P.C., Stell G., Phys. Rev. Lett., 1970, 24, No. 23, 1284; doi 10.1103/PhysRevLett.24.1284

32. Stell G., Hemmer P.C., J. Chem. Phys., 1972, 56, No. 9, 4274; doi 10.1063/1.1677857.

33. Young D.A., Alder B.J., Phys. Rev. Lett., 1977, 38, No. 21, 1213; doi 10.1103/PhysRevLett.38.1213

34. Jagla E.A., J. Chem. Phys., 1999, 111, No. 19, 8980; doi 10.1063/1.480241.

35. Rzysko W., Pizio O., Patrykiejew A., Sokołowski S., J. Chem. Phys., 2008, 129, No. 12, 124502; doi $10.1063 / 1.2970884$

36. Buldyrev S.V., Malescio G., Angell C.A., Giovambattista N., Prestipino S., Saija F., Stanley H.E., Xu L., J. Phys.: Condens. Matter, 2009, 21, No. 50, 504106; doi 10.1088/0953-8984/21/50/504106

37. Klein W., Gould H., Ramos R.A., Clejan I., Melcuk A.I., Physica A, 1994, 205, No. 4, 738; doi 10.1016/0378-4371(94)90233-X

38. Norizoe Y., Kawakatsu T., Europhys. Lett., 2005, 72, No. 4, 583; doi 10.1209/epl/i2005-10288-6

39. Norizoe Y., Kawakatsu T., J. Chem. Phys., 2012, 137, No. 2, 024904; doi 10.1063/1.4733462

40. Camp P.J., Phys. Rev. E, 2003, 68, No. 6, 061506; doi 10.1103/PhysRevE.68.061506

41. Malescio G., Pellicane G., Phys. Rev. E, 2004, 70, No. 2, 021202; doi 10.1103/PhysRevE.70.021202

42. Glaser M.A., Grason G.M., Kamien R.D., Kosmrlj A., Santangelo C.D., Ziherl P., EPL, 2007, 78, No. 4, 46004; doi $10.1209 / 0295-5075 / 78 / 46004$

43. Mladek B.M., Gottwald D., Kahl G., Neumann M., Likos C.N., J. Phys. Chem. B, 2007, 111, No. 44, 12799; doi 10.1021/jp074652m

44. Fornleitner J., Kahl G., EPL, 2008, 82, No. 1, 18001; doi 10.1209/0295-5075/82/18001

45. Shin H.M., Grason G.M., Santangelo C.D., Soft Matter, 2009, 5, No. 19, 3629; doi 10.1039/B904103F

46. Dotera T., Oshiro T., Ziherl P., Nature, 2014, 506, No. 7487, 208; doi 10.1038/nature12938

47. Likos C.N., Lang A., Watzlawek M., Lowen H., Phys. Rev. E, 2001, 63, No. 3, 031206; doi 10.1103/PhysRevE.63.031206

48. Mladek B.M., Gottwald D., Kahl G., Neumann M., Likos C.N., Phys. Rev. Lett., 2006, 96, No. 4, 045701; doi $10.1103 /$ PhysRevLett.96.045701

49. Klix C.L., Royall C.P., Tanaka H., Phys. Rev. Lett., 2010, 104, No. 16, 165702; doi 10.1103/PhysRevLett.104.165702

50. Batten R.D., Huse D.A., Stillinger F.H., Torquato S., Soft Matter, 2011, 7, No. 13, 6194; doi 10.1039/c0sm01380c.

51. Liu Y.H., Chew L.Y., Yu M.Y., Phys. Rev. E, 2008, 78, No. 6, 066405; doi 10.1103/PhysRevE.78.066405 
52. Malescio G., Pellicane G., Nat. Mater., 2003, 2, No. 2, 97; doi $10.1038 /$ nmat820

53. Sciortino F., Tartaglia P., Zaccarelli E., J. Phys. Chem. B, 2005, 109, No. 46, 21942; doi 10.1021/jp052683g

54. Zaccarelli E., J. Phys.: Condens. Matter, 2007, 19, No. 32, 323101; doi 10.1088/0953-8984/19/32/323101

55. Allen M.P., Tildesley D.J., Computer simulations of liquids, Oxford University Press, Oxford, 1987.

56. Baumketner A., J. Phys. Chem. B, 2014, 118, No. 50, 14578; doi 10.1021/jp509213f

57. Mossa S., Sciortino F., Tartaglia P., Zaccarelli E., Langmuir, 2004, 20, No. 24, 10756; doi 10.1021/la048554t

58. De Gennes P.G., Scaling Concepts in Polymer Physics, Cornell University Press, New York, 1979.

59. Dobnikar J., Fornleitner J., Kahl G., J. Phys.: Condens. Matter, 2008, 20, No. 49, 494220;

doi $10.1088 / 0953-8984 / 20 / 49 / 494220$

\title{
Рівноважні кластери в розчинах колоїдів, що взаємодіють через потенціал з локальним мінімумом
}

\author{
А. Баумкетнер ${ }^{1}$, В. Kaй² \\ ${ }^{1}$ Інститут фізики конденсованих систем НАН України, вул. І. Свєнціцького, 1, 79011 Львів, Україна \\ 2 дослідний центр обчислювальних наук у м. Пекін, 1000094 Пекін, Китай
}

\begin{abstract}
В простих колоїдних розчинах кластерами називаються мультимери, що виникають внаслідок асоціації мономерів і співіснують з ними в динамічній рівновазі. Існують два типи потенціалів, що ведуть до утворення кластерів: а) потенціали з глобальним мінімумом, що можуть виникнути, зокрема, внаслідок неповної компенсації притягальної взаємодії на коротких відстанях та відштовхувальної взаємодії на довгих відстанях, б) повністю відштовхувальні взаємодії, які демонструють м'яке “плече”. За допомогою комп'ютерних симуляцій в даній роботі показано, що потенціали з локальним мінімумом і відштовхувальним хвостом теж мають здатність утворювати рівноважні кластери. Хоча вони не належать до жодного з перелічених вище класів, аналіз показує, що такі потенціали мають з ними певні спільні характеристики. При малій густині колоїдів кластери, утворені новими потенціалами, видовжені подібно до кластерів в суто відштовхувальних системах. При великій густині, нові кластери компактні, як це спостерігається в системах з глобальним потенціальним мінімумом. Отже, нові потенціали слугують тим містком, який поєднує два відомі класи потенціалів, що ведуть до утворення кластерів в колоїдних розчинах.
\end{abstract}

Ключові слова: колоїди, кластери, локальний мінімум, відштовхувальний потенціал, комп'ютерні симуляції 